\section{Endoscopic Ligation for Bleeding Rectal Varices in a Child with Primary Extrahepatic Portal Hypertension}

A 13-year-old boy was admitted to our hospital because of rectal bleeding. He was $137 \mathrm{~cm}$ tall and weighed $37.5 \mathrm{~kg}$ (like an average eight-year-old Japanese boy). He had been diagnosed as having primary extrahepatic portal vein obstruction (EHPVO) when he was three years old. He underwent sclerotherapy for esophageal varices four times between two to four years after diagnosis of EHPVO. Sigmoidoscopy revealed three huge, tortuous rectal varices arranged perpendicular to the Houston rectal fold and extending $10 \mathrm{~cm}$ from the anus (Figure 1). The red bleeding spot, similar to the red color sign of esophageal varices, was found in the ridge of one varix which had a white color. was thin and protruding. Endoscopic variceal ligation (EVL) was performed on three rectal varices. Ligation was repeated from the proximal to the distal ends, and two bands were placed on two blue varices and three bands were placed on one white varix. No immediate complications occurred. A repeat sigmoidoscopy three days later showed bleeding from the edge of the post-EVL ulcerations that was the result of the detachment of the band and the entrapped mucosa (Figure 2). However, sigmoidoscopy 20 days later showed complete resolution of the varices and healing ulcers without any evidence of bleeding. In addition, sigmoidoscopy 72 days later showed multiple scars of post-EVL ulcers (Figure 3 ). There have been no recurrent bleeding

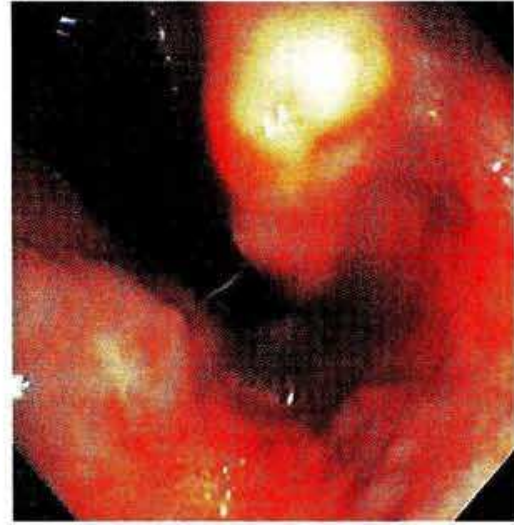

Figure 1 Retroflex endoscopic view of rectum before ligation. Markedly dilated varices can be seen in the rectum. internal hemorrhoids are not seen in the anus and rectum

episodes over a follow-up period of 13 months.

It is well known that endoscopic sclerotherapy is an effective method for controlling bleeding from rectal varices $[1,2]$. However, a case where sclerotherapy was ineffective has been reported [3]. Two reports $[4,5]$ so far have shown EVL therapy to be effective in controlling bleeding from rectal varices. EVL for rectal varices appears to be a safe and effective therapy, even in children.

Y. Uno', A. Munakata', A. Ishiguro',

S. Fukuda', M. Sugai ${ }^{2}$. H. Munakata 2

' First Dept. of Internal Medicine,

Hirosaki University School of Medicine,

Hirosaki, Aomori, Japan

${ }^{2}$ Dept. of Pediatric Surgery,

Hirosaki University School of Medicine, Hirosaki, Aomori, Japan

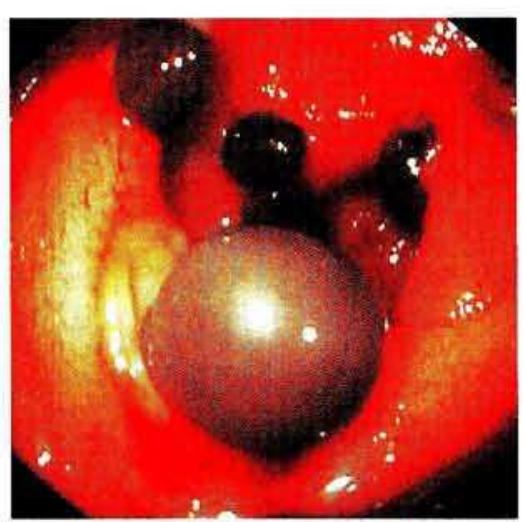

Figure 2 At three days after ligation black necrotic mucosa with hemorrhage can be seen at the site of ligation

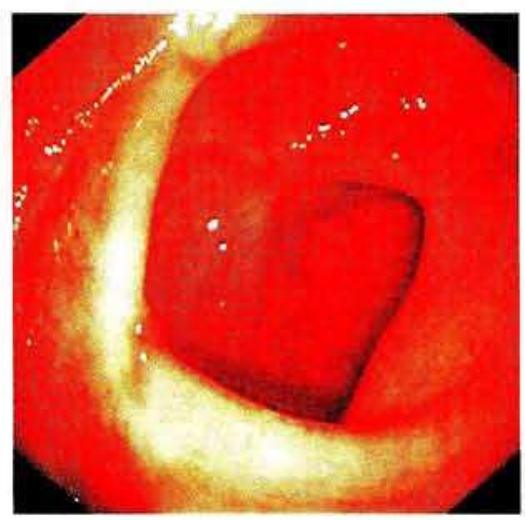

Figure 3 At 72 days after ligation multiple scars can be seen in the rectum 


\section{S108 Unusual Cases and Technical Notes}

\section{References}

1. Wang M, Desigan G, Dunn D. Endoscopic sclerotherapy for bleeding rectal varices. Am J Gastroenterol 1985; 80: $779-80$

2. Weiserbs DB, Zfass AM, Messmer J. Control of massive hemorrhage from rectal varices with sclerotherapy. Gastrointest Endosc 1986; 32: 419-21

3. Herman BE, Baum S, Denobile J, et al. Massive bleeding from rectal varices. Am J Gastroenterol 1993; 88: 939-42
4. Levine J, Tahiri A, Banerjee B. Endoscopic ligation of bleeding rectal varices. Gastrointest Endosc 1993; 39: $188-90$

5. Kojima T, Onoda M, Tajiri T, et al. A case of massive bleeding from rectal varices treated with endoscopic variceal ligation. Jpn J Gastroenterol 1996; 93: $114-9$
Corresponding Author

Y. Uno, M.D.

First Dept. of Internal Medicine

Hirosaki University School

of Medicine

5 Zaifu-cho, Hirosaki

036 Aomori

Japan

Fax: $\quad+81$ (172) 375946

E-mail: sfukuda@cc.hirosaki-u.ac.jp 